\title{
Capas de refuerzo con hormigón adheridas como alternativa para la rehabilitación de pavimentos asfálticos
}

\section{Bonded concrete overlays as a rehabilitation alternative for asphalt pavements}

\author{
Marianela Espinoza Alfaro \\ Universidad de Costa Rica, Costa Rica \\ mespinoza091@gmail.com
}

Fecha de recepción: 29 de febrero de 2016 / Fecha de aprobación: 10 de marzo de 2016

\section{RESUMEN}

Este trabajo tiene como objetivo dar a conocer las consideraciones básicas al momento de diseñar una capa de refuerzo con hormigón como alternativa para la rehabilitación de pavimentos existentes. Primero se evalúa la condición del pavimento; determinando el tipo de deterioro, las reparaciones previas, así como la necesidad de preparación de la superficie; luego se identifica la posible aplicación de este tipo de solución. Las capas de refuerzo adheridas son una estrategia sustentable para preservar y extender la vida de los pavimentos que pueden usarse como rehabilitación menor, cuyo propósito principal es adicionar capacidad estructural y eliminar daños superficiales en los pavimentos existentes; esto mediante una adecuada unión entre la capa de refuerzo a colocar y el pavimento. Costa Rica actualmente cuenta con un elevado deterioro en los pavimentos que conforman la mayor parte de las carreteras de la red vial; donde muchas veces - por falta de conocimiento- se eluden alternativas factibles de solución. Por ello se realiza una investigación sobre la opción de uso de capas de refuerzo adheridas, considerando los requerimientos para ser aplicada, así como sus metodologías de diseño. A partir de la información recopilada se pretende brindar a los ingenieros viales un documento que facilite la decisión de optar por una solución ante problemas comunes encontrados en los pavimentos existentes, para lograr ampliar el conocimiento en esta área y permitir su uso en la infraestructura vial y el servicio brindado al usuario.

PALABRAS CLAVE: Capas de refuerzo, rehabilitación, pavimento, deterioro, refuerzo, hormigón.

\section{ABSTRACT}

The main objective of this study, is to present basic considerations while designing a concrete overlay as a rehabilitation alternative for existing pavements. First, the pavement condition has to be evaluated by determining the type of damage, previous repairs, and the need for surface preparation; then it is necessary to evaluate the possibilities for the application of this technique. Bonded concrete overlays are a sustainable strategy to preserve and extend the life of pavements that can be used as minor rehabilitation, which its main purpose is to add structural capacity and to eliminate surface damage on existing pavements by creatingan adequate bond between the concrete overlay and the pavement structure. High deterioration levels have been identified on pavement structures in Costa Rica, which make up most of the roads in the national road network, where quite often feasible solution alternatives are not considered due to a lack of knowledge. Therefore, this literature review focuses on the use of bonded concrete overlays, considering the construction requirements, as well as the design methodologies. This paper intends to provide road engineers with a reference document to help in deciding the most adequate solution to common problems found on existing pavements, in order to increaseknowledge in this area, to encourage the use of new techniques to improve road infrastructure, therefore improve the quality of service provided to the user.

KEYWORDS: Overlays, rehabilitation, pavement deterioration, reinforcement, concrete. 


\section{INTRODUCCIÓN}

La rehabilitación en pavimentos es un tema que ha formado parte de la ingeniería a lo largo de la historia. Según el programa de investigación en carreteras de Estados Unidos NCHRP (1994), los inicios de capas de refuerzo datan alrededor del año 1913, avanzando su aplicación hasta 1981, donde surge el nuevo concepto de adherencia entre la carpeta asfáltica existente y la nueva capa a colocar. Sin embargo, su aceptación de uso fue hasta años después cuando el aumento en la demanda, producto del rápido deterioro de las carreteras, llevó a diferentes entidades a optar por la alternativa de capas de refuerzo con hormigón, como una solución clave con resultados satisfactorios y a costos equilibrados de financiamiento.

Este método de rehabilitación tiene el propósito de extender la vida del pavimento asfáltico con la adición de un espesor de hormigón, logrando aumentar la integridad estructural, proteger contra efectos ambientales y mejorar la calidad de la estructura, así como acelerar la construcción y reducir costos. Entre los tipos de capas adheridas se encuentran desde las convencionales (mayores a $200 \mathrm{~mm}$ de espesor), hasta las delgadas (100mm-200mm). Además, existe un nuevo concepto de capas ultradelgadas con espesores menores a 100mm (Rasmussen y Rozychi, 2004). Estos espesores son justificados con el uso de hormigón de alta calidad, resistencia alta, menor espaciamiento entre juntas y una correcta adherencia con el pavimento existente. La asociación de pavimentos de concreto de Estados Unidos ACPA (1992), dio seguimiento de uso de estas últimas (capas ultradelgadas) en Estados Unidos, siendo en 1991 en la ciudad de Louisville, Kentucky, donde se construyó el primer proyecto con capas ultradelgadas (Cole y Mohsen, 1993). También en Europa se cita el primer proyecto de construcción con este tipo de solución, y otros países que han optado por su uso son: Canadá, México, Brasil, República de Corea, Japón, Francia, Austria y Países Bajos.

Datos documentados por el explorador nacional de capas de refuerzo con hormigón (National Concrete Overlay Explorer) de la ACPA revelan que entre 1980 y 2010 en Estados Unidos se construyeron cinco veces más proyectos de capas de refuerzo con hormigón por década que los construidos en las seis décadas anteriores. El desarrollo de un procedimiento de diseño mecanicista representó otro paso importante en el avance de esta técnica (Mack, Cole y Mohsen, 1993).

Según el Manual Centroamericano para Diseño de Pavimentos (Coronado, 2002), para el 2001 Costa Rica contaba con $65 \mathrm{~km}$ de pavimentos deconcreto hidráulico. Ha incrementado su uso por la razón de nivelar los precios de mercado y realizar inversiones (equipo, tecnología) por parte de empresas privadas; no obstante, el uso de capas de refuerzo delgadas y ultradelgadas aún se encuentra sin explorar a fondo. A pesar de proyectos exitosos que se han realizado a nivel mundial, muchas entidades dudan de su escogencia en la toma de decisión, lo cual puede deberse a una mala conceptualización de las técnicas mencionadas. Es por ello que mediante este artículo se pretende mostrar los conceptos fundamentales a considerar ante la necesidad de rehabilitación de un proyecto de carretera, ampliando el conocimiento y facilitando la escogencia de la opción óptima.

El artículo se divide en tres partes: en la primera parte se detalla los tipos y características de las capas de refuerzo, comparando opciones de forma práctica y entendible; la segunda parte reúne los criterios de selección de acuerdo a las condiciones del pavimento existente; $y$ la tercera parte detalla las consideraciones básicas de diseño, así como las metodologías disponibles que facilitan su aplicación.

\section{PARTE I}

Al hablar de alternativas para la rehabilitación de pavimentos, es importante conocer los principales tipos de capas de refuerzo, las cuales se clasifican en adheridas y no adheridas. La primera de ellas se usa cuando se desea eliminar deterioros superficiales y el pavimento existente presenta una buena condición estructural. En este tipo de capa es esencial una buena adherencia, permitiendo que ambas capas trabajen como un pavimento monolítico, por lo que es necesario preparar la superficie sobre la que se va a colocar la nueva capa. La segunda opción corresponde a una rehabilitación mayor dondela preparación previa es menor y proporciona una vida de diseño mayor a una capa con asfalto; su propósito es restaurar la capacidad estructural en una condición de deterioro de moderada a severa. En este documento se dará énfasis en la primera opción descrita, correspondiente a capas adheridas.

La Figura 1 muestra esquemáticamente la división de los tipos de capas de refuerzo con hormigón.

En la Figura 2 semuestra las condiciones del pavimento a lo largo de su vida. Las zonas de mantenimiento preventivo, rehabilitaciones menores y rehabilitaciones mayores, se muestran separadas por rangos donde se puede utilizar refuerzos con o sin adherencia. En la figura se observa una elección de capas adheridas cuando la condición del pavimento existente se encuentra de regular a buena, y en caso de tener una condición de pobre a regular, se puede optar por una aplicación de capa no adherida. 


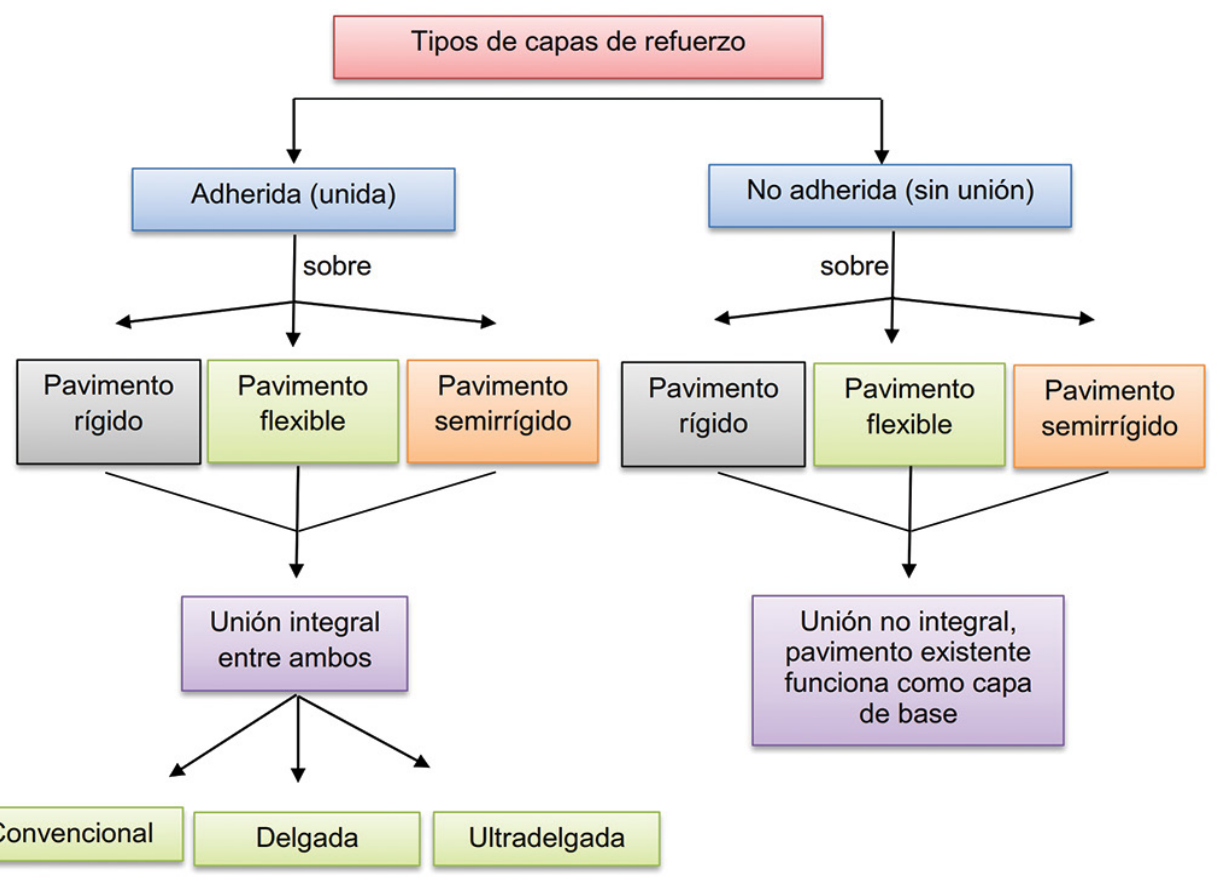

Figura 1. Tipos de capas de refuerzo

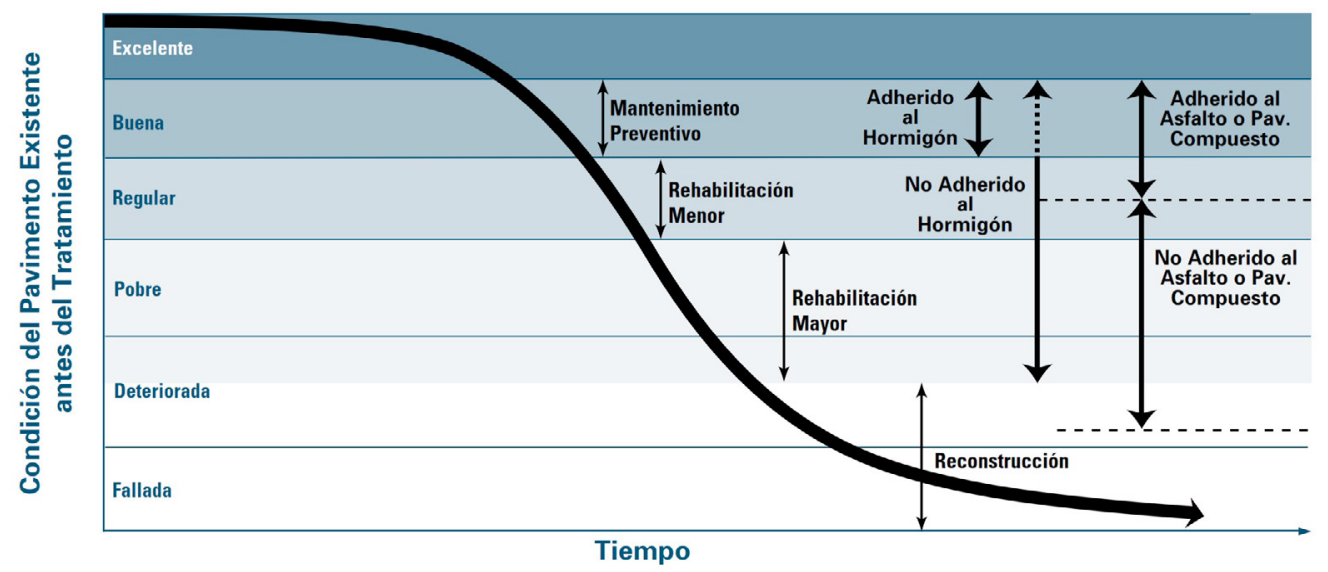

Figura 2. Soluciones típicas con hormigón adherido y no adherido en diferentes etapas de vida de servicio del pavimento. (Harrington y Fick,2014).

Las capas adheridas pueden dividirse en varios tipos, como lo son convencionales, delgadas y ultradelgadas (Han, 2005), para lo cual se muestra la Tabla 1, donde se comparan las ventajas y desventajas respectivamente. Investigación realizada (Han, 2005), señala que las capas con hormigón delgadas y ultradelgadas pueden presentar costos iniciales mayores que una capa de asfalto, sin embargo, durante el ciclo de vida de la estructura, permiten extender la vida del pavimento de dos a tres veces en comparación con las capas de asfalto, requiriendo menos intervenciones para mantenimiento. Al procurar una adecuada adherencia con la adición de fibras (acero, sintéticas) al hormigón, se pueden obtener mejores beneficios. Esta adherencia puede ser percibida desde dos ángulos: en primer lugar, las condiciones y cinética de unión de dos materiales, de acuerdo a los mecanismos a utilizar, y en segundo lugar la medida de la magnitud de adición, comúnmente expresada en esfuerzo o energía requerida para separar los dos materiales (Silfwerbrand, Beushausen y Courard, 2011).

El esquema mostrado en la Figura 3, basado en información publicada en: Síntesis de prácticas actuales para capas delgadas y ultradelgadas en Minnesota (Han, 2005), muestra las características básicas ante la escogencia del tipo de capa delgada y ultradelgada. 


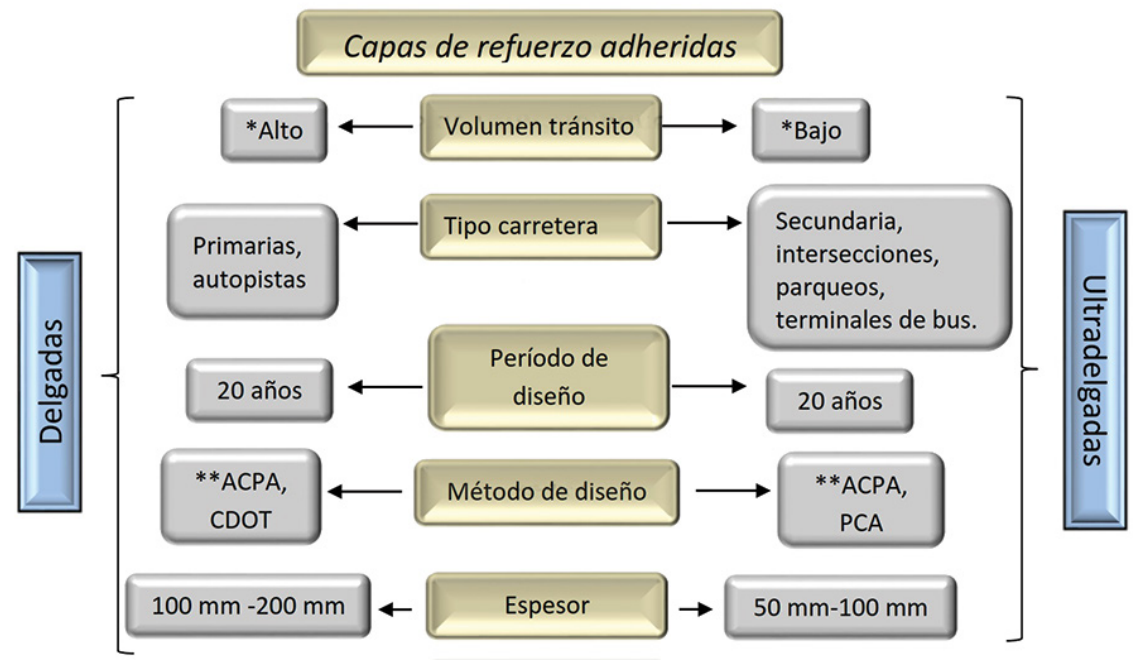

* Alto: tránsito promedio diario anual $>10000$ vpd Bajo: tránsito promedio diario anual<400 vpd **Asociación Americana de Pavimentos de Concreto Departamento de Transporte de Colorado Asociación de Cemento Portland

Figura 3. Esquema comparativo: tipos capas de refuerzo adheridas (Han, 2005). Nota: modificado por el autor.

Tabla 1. Tipos de capas adheridas (Han, 2005). Nota: modificado por el autor.

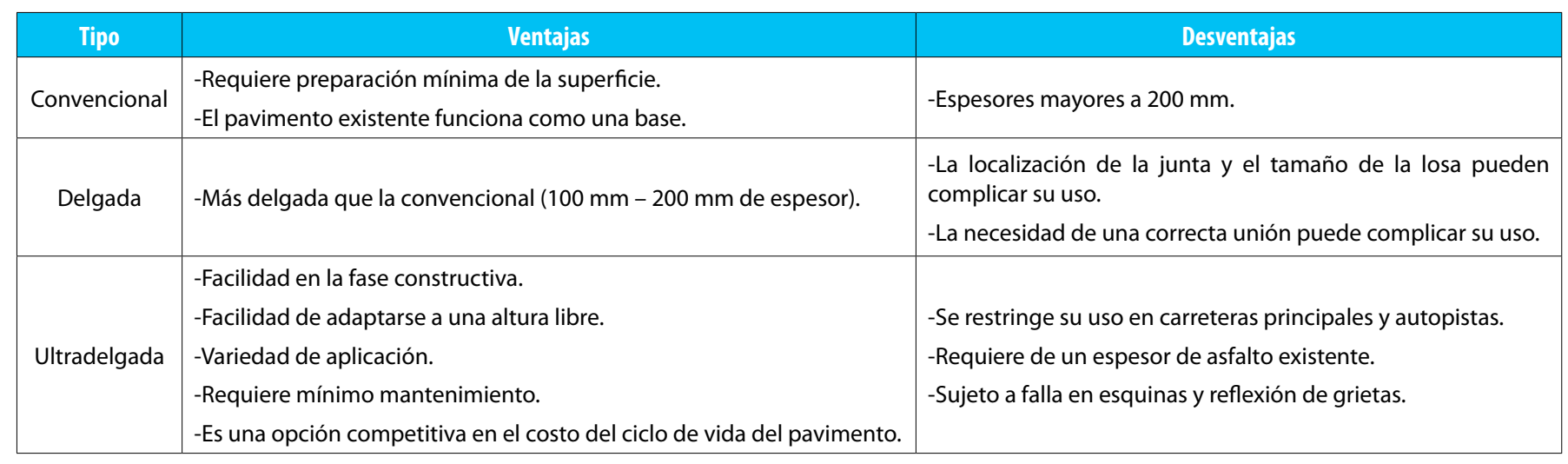

\section{PARTE II}

\section{Criterios de selección}

Para elegir la opción que mejor se adapte a las necesidades del proyecto se debe de considerar algunos criterios fundamentales, entre ellos la evaluación del pavimento, que genere información importante sobre sus condiciones (estado, capacidad y limitaciones). En la Figura 4 se muestra una secuencia de las etapas para la evaluación, finalizando en un reporte o informe final completo que facilite el posterior diseño.

Se debe buscar archivos en la base de datos del proyecto en estudio donde se muestre el historial del pavimento existente, permitiendo considerar los factores que fueron utilizados para su diseño y en la elaboración de informes, planos, especificaciones, así como estudios, auscultaciones y rehabilitaciones efectuadas. Junto a esta información, también se debe considerar las cargas de tránsito proyectado y la vida de diseño del refuerzo.

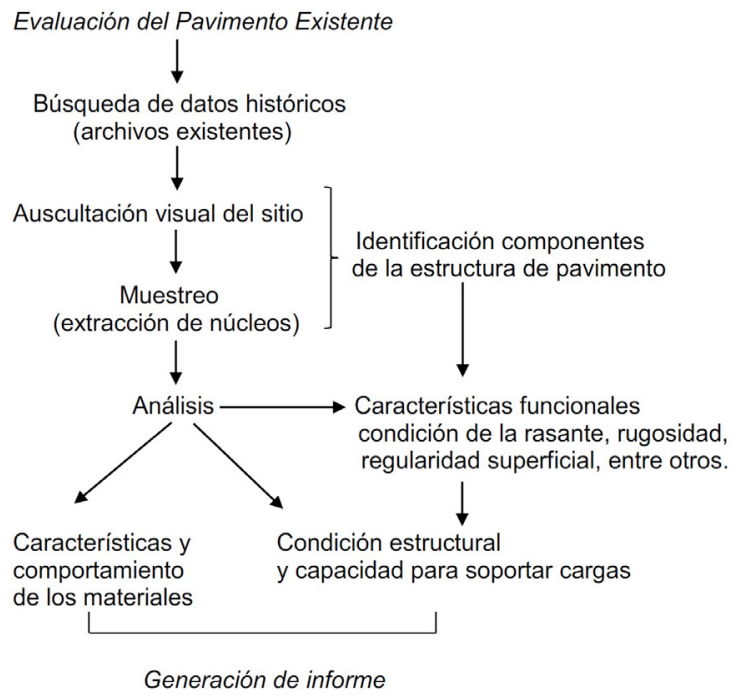

Figura 4.Esquema para Evaluación del pavimento existente 


\section{Auscultación visual}

En esta etapa de la evaluación se determina los deterioros observables en el pavimento, los cuales pueden estar asociados a cargas de tránsito, un drenaje deficiente, clima, deficiencias a nivel constructivo y materiales, siendo comunes los que se enumeran a continuación:

1. Ahuellamiento: debido a asentamientos de los materiales bajo un tránsito pesado y canalizado.

2. Fatiga: grietas interconectadas formando bloques angulosos, producto de cargas repetidas del tránsito.

3. Grietas en bloque: dividen el pavimento en bloques rectangulares, causadas principalmente por contracción y oxidación de la mezcla asfáltica.

4. Exudación: subida de los excedentes de material bituminoso a la superficie de la calzada.

5. Agrietamiento en los bordes: corresponden a grietas continuas que cruzan el borde del pavimento, adyacente al espaldón.

6. Grietas longitudinales: paralelas a la línea central del pavimento, localizadas dentro del carril.

7. Grietas transversales: perpendiculares a la línea de centro del pavimento.

La publicación (Manual de identificación de deterioros) de la Administración Federal de Carreteras (2003) puede resultar útil para definir los diferentes tipos de deterioro encontrados. En Costa Rica el método de auscultación francesa VIZIR desarrollado por el Laboratorio Central de Puentes y Calzadas de Francia en 1972, en su traducción al español (Autret y Brousse,1992), utilizado por el Ministerio de Obras Públicas y Transportes de Costa Rica desde 1995, permite la evaluación de la condición del pavimento, mediante el índice de deterioro superficial (IS), que varía desde 1 hasta 7 , según la condición, clasificando los daños en Tipo A y Tipo B, dependiendo de si están asociados a la capacidad estructural o no. Independiente de la metodología a utilizar, la cual puede variar según el sitio y proyecto específico, la clave en esta etapa es identificar adecuadamente los deterioros presentes, su severidad, las causas que dieron origen y la medida de rehabilitación más adecuada.

De acuerdo con el Informe de Evaluación de la Red Vial Nacional Pavimentada de Costa Rica 2014-2015, realizada por el Laboratorio de Materiales y Modelos Estructurales de la Universidad de Costa Rica (LanammeUCR, 2015), detalla que $308,98 \mathrm{~km}$ de la Red Vial Nacional evaluada, requiere de actividades de rehabilitación menor y mayor, por lo que amerita planificar la intervención a realizar y ajustar el presupuesto optimizando las inversiones. Es importante analizar este tipo de alternativa para determinar la posibilidad de su aplicación.

\section{Muestreo}

Una vez finalizado la inspección visual, resulta importante considerar la extracción de núcleos con el fin de obtener los espesores de la estructura presente, el tipo y materiales de cada una de las capas, la determinación del valor de soporte y demás características que expliquen el comportamiento del pavimento. Este paso es esencial si se está considerando la opción de una capa delgada o ultradelgada, en donde es crítico evaluar la adherencia entre capas, para identificar cuáles de ellas se encuentran desprendidas, y así determinar si esa capa de asfalto proveerá una base adecuada sobre la que se colocará la capa de refuerzo con hormigón. En caso de que el pavimento existente se encuentre en malas condiciones y haya presencia de tránsito pesado, se puede optar por ensayos opcionales como deflectometría, ensayos de rugosidad y fricción superficial.

\section{Análisis}

Al recolectar la información proveniente de datos históricos, inspección visual, muestreos y demás, se debe tabular y graficar los resultados obtenidos, con el fin de analizar, comparar y discutir el comportamiento funcional y estructural del pavimento, con el fin de que el ingeniero pueda escoger la opción de rehabilitación que mejor se adapte a las necesidades del pavimento.

\section{Informe final}

El informe constituye una síntesis de la investigación previa realizada, donde se reúne los aspectos generales de la información recolectada y el análisis de resultados que facilitan al ingeniero vial la elección de su alternativa de capa de refuerzo a diseñar. Una vez realizada la evaluación se debe de definir los criterios que indiquen la mejor selección. Para ello se puede dividir en condiciones buenas, regulares, malas y deterioradas, como lo expone la Guía para Capas de Refuerzo con Hormigón (Harrington y Fick, 2014).

Buena condición: Pavimento sano, únicamente requiere incrementar su capacidad estructural, mejorar sus características superficiales, remover deterioro superficial y/o mejorar propiedades de reflectancia.

Condición regular: El pavimento está estructuralmente sano, pero tiene deterioro superficial menor como baches, agrietamiento térmico y grietas en bloque. Se recurre a verificar desprendimientos en las muestras así como ondulaciones en la rasante. 
Mala condición: Hay presencia de deterioro mayor a los dos casos anteriores; es posible observar agrietamiento por fatiga, ahuellamiento, ondulaciones, desprendimientos y desgaste superficial.

Condición deteriorada: Similar a la mala condición y con un desgaste superficial significativo, expansión térmica, desprendimientos y deterioro estructural.

Ante las descripciones expuestas para poder utilizar capas de refuerzo adheridas delgadas y/o ultradelgadas se debe verificar si con la aplicación de fresado y reparaciones puntuales menores se pueden resolver las deficiencias, sin alterar componentes de la vía como gálibos (claro vertical libre), espaldón, barreras de seguridad, entre otros. Esta evaluación es sumamente importante para asegurarse de que existe una capacidad estructural que soporte la aplicación de cargas, determinar si requiere fresado y seleccionar el espesor requerido.

\section{PARTE III}

En el diseño de la capas es necesario definir una serie de variables que van a depender de las condiciones propias en las que se desarrolle el proyecto. En este aspecto es sumamente importante conocer la vida de diseño que se desea, puesto que esta variable va a influir en la escogencia del espesor y las reparaciones requeridas, y por ende en el costo de la capa de refuerzo.

\section{Factores a considerar en diseño}

Enla Figura 5 se muestra los principales factores que afectan el desempeño del pavimento. Es clave una definición, análisis y

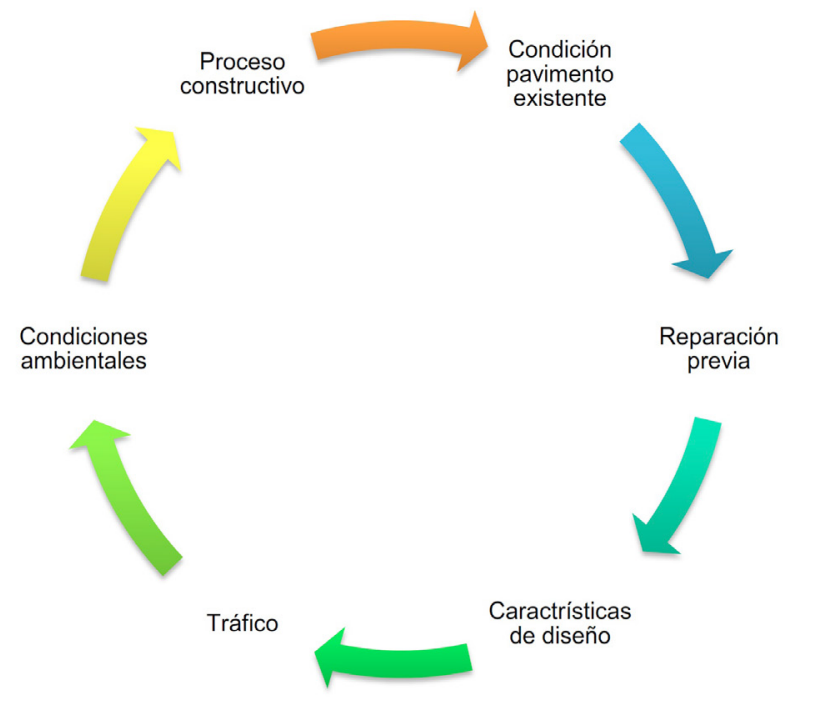

control de cada uno de ellos a fin de obtener resultados precisos y poder predecir de manera realista el comportamiento del diseño propuesto.

\section{Evaluación del pavimento existente (tipo, severidad y grado de deterioro)}

En esta etapa como se mencionó previamente, es fundamental una evaluación acertada de la condición existente. Estudios recientes (NCHRP, 2004), indican que la susceptibilidad de la mezcla asfáltica a la deformación permanente es un factor significativo en el desempeño de las capas de refuerzo delgadas y ultradelgadas. La medición de la severidad puede medirse por niveles; de acuerdo a Manual de identificación de deterioros (Miller y Bellinger, 2003), donde los niveles detectados son: bajo, moderado y alto, cada uno de ellos para el tipo de deterioro. También la evaluación visual de la condición del pavimento se puede realizar usando el Índice de Condición del Pavimento o PCI, por sus siglas en inglés, el cual es un índice que varía entre 0 y 100 , siendo 0 para pavimentos fallados (mal estado) y 100 para pavimentos en perfecto estado (Norma ASTM D6433-11, 2011). Una adecuada evaluación permitirá al ingeniero considerar la necesidad o no de reparación previa y el tipo respectivo.

\section{Reparaciones previas}

La preparación de la superficie sobre la que se colocará la nueva capa de pavimento es esencial, debe de proveer las condiciones adecuadas que permitan una óptima adherencia entre ambas capas, propiciando una reducción tanto de las tensiones bajo la aplicación de la carga, como de los posibles levantamientos de los bordes y esquinas de las losas. En la Figura 6 se muestra un ejemplo de reparación previa.

Una de las reparaciones más comunes es el fresado (obtención de nuevo perfil longitudinal y transversal del pavimento), que permite suavizar las distorsiones de la superficie y establecer una base adecuada para la nueva capa a colocar. Es recomendable mantener un mínimo de espesor en la estructura existente; según recomendación de la ACPA se debe mantener $75 \mathrm{~mm}$, previendo errores en la evaluación del espesor de capa que puedan llevar a gastos significativos; principalmente porque si queda muy poco espesor de pavimento que sirva como capacidad estructural, será necesario aumentar el grosor de la capa de refuerzo. El costo es un factor significativo y habitual en la determinación del grado de reparación; cualquier adicional puede resultar en un espesor reducido, debido a que el costo del proyecto se mantiene fijo.

Figura 5. Factores a considerar en el diseño 


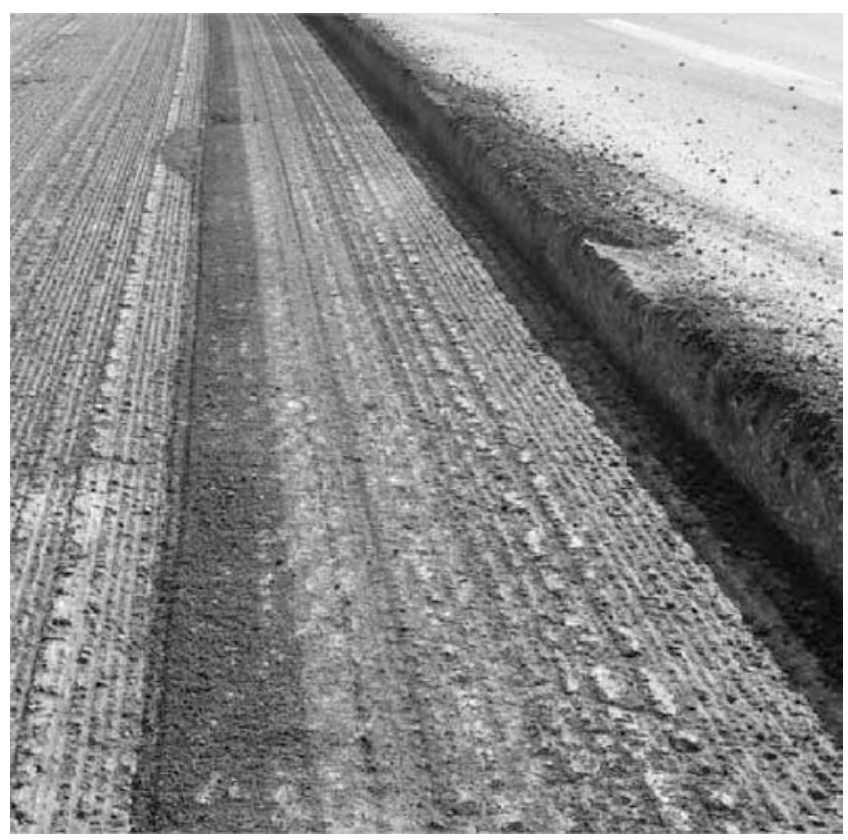

Figura 6. Ejemplo de reparación previa: fresado. (Rasmussen y Rozychi, 2004)

\section{Tráfico (ejes equivalentes)}

La predicción de las solicitaciones del tránsito es un factor fundamental en el diseño de cualquier estructura de pavimento. El número de ejes equivalentes que debe soportar durante su vida útil prevista determinará la clase de capa a usar. Nuevas tendencias de caracterización del tráfico para metodologías mecánico-empíricas definen aspectos como: espectros de distribución del número de ejes (peso y tipo), distribución estacional del tráfico, el nivel de crecimiento del tránsito (requiriendo factores de ajuste), hora del día, temperatura.

\section{Características de diseño}

Una característica fundamental en el diseño es el espesor de la losa; este va a depender de una serie de factores como el tráfico previsto, las propiedades del pavimento existente y del hormigón a colocar, la transferencia de cargas, etc.

Se debe tener precaución de que una vez realizado el fresado (de ser necesario) y la colocación de la capa, el espesor sea el adecuado y no altere el drenaje ni genere problemas de altura libre entre estructuras. Para capas menores de $100 \mathrm{~mm}$ con espaciamiento de $0,9 \mathrm{~m}$, el espesor de la carpeta existente puede variar entre $60 \mathrm{~mm}$ y $120 \mathrm{~mm}$, según el artículo investigativo de factibilidad (Mampearachchi, Kosgolla y Tia, 2012). Cuando se presentan problemas para elevar la cota de la estructura existente, ya sea por escasez de gálibo bajo los puentes, dificultad de acceso a propiedades o para evitar intervenir en los bordes de la calzada y barreras de seguridad, se puede optar por la abertura de una caja en cuyo interior se vierte el hormigón de refuerzo, procurando que el espesor de pavimento dentro del cajeado, constituido por dicho pavimento y el existente, tenga capacidad estructural suficiente para soportar el tráfico del proyecto (Abascal, 2013).

Las juntas también forman parte del diseño, en el caso de capas delgadas y ultradelgadas se caracterizan por tener espaciamientos cortos de las juntas, reduciendo de esta manera las tensiones. Esta separación usualmente es de 12 a 18 veces el espesor (Rasmussen y Rozychi., 2004). En este tipo de capas donde la transferencia de cargas se basa en la trabazón de agregados, con una buena graduación se puede reducir la separación. Sin embargo el uso de varillas de anclaje y transferencia de carga se han utilizado con éxito. El diseñador debe procurar comprobar la ubicación del trayecto a seguir por la llanta, para que las juntas longitudinales se coloquen lejos, a fin de evitar posibles grietas. Estudios realizados por el Departamento de Transportes de Minnesota (MnDOT, 2013), han demostrado mejores comportamientos cuando se sellan las juntas (Rasmussen y Rozychi., 2004).

La relación longitud-ancho de la losa es otro factor a determinar, el cual de acuerdo a estudios previos se recomienda tener una proporción de 1,25 (ACPA, 1998), prefiriendo el uso de losas cuadradas con dimensiones pequeñas, en un rango de $0,9 \mathrm{~m}$ y 2,4m (Rasmussen y Rozychi., 2004), con el fin de disminuir posibles movimientos diferenciales entre capas y efectos de alabeo. También es importante considerar el efecto del módulo elástico; se ha analizado que el esfuerzo principal y el esfuerzo cortante en la interface crecen significativamente al aumentar el módulo de la capa de hormigón, por lo que es necesario asegurar una buena adherencia entre capas. Estos esfuerzos en el hormigón tienden a decrecer cuando el módulo elástico de la carpeta existente aumenta, de manera que se debe procurar mantener un módulo suficiente en el asfalto, para generar una buena interacción junto a la capa de hormigón. En el caso de la base, el módulo tiene un comportamiento similar, en donde el esfuerzo principal aumenta cuando la rigidez en la base disminuye. En el caso de la subrasante el efecto del módulo sobre los esfuerzos en la capa de hormigón no es significante (Mampearachchi et al., 2012).

\section{Materiales}

En cualquier diseño, los materiales a utilizar deben de responder a ciertas propiedades básicas. Los agregados constituyen la mayor parte del espesor a colocar, y sus propiedades pueden intervenir en el desempeño de la capa de refuerzo, de manera que se debe controlar el tamaño máximo de los agregados. Para ello se debe asegurar una adecuada graduación (combinación de tamaños) que permita lograr la trabazón entre ellos, lo cual es necesario 
en el caso de capas delgadas y ultradelgadas. Otra propiedad es el coeficiente de expansión térmica, el cual si es muy alto, puede provocar esfuerzos mayores sobre la losa y generación de grietas. Se recomienda que el coeficiente de expansión del agregado grueso en la capa de refuerzo no sea mayor al presente en el pavimento existente (Trevino, McCullough y Fowler, 2003).

Otro material es el cemento, el cual puede afectar el comportamiento y rendimiento, por la variación de las propiedades en función del tiempo, principalmente en edades tempranas. En la mezcla, la relación agua-cemento es crítico en la predicción de la fuerza total y su durabilidad. Las fibras son otro material que ha aumentado su uso;estudios muestran que la adición de fibras al hormigón incrementa significativamente su desempeño, antes y después de iniciada la grieta en la losa (Bordelon y Roesler, 2012), mejoran su tenacidad y resistencia residual.El uso de las fibras pueden beneficiar particularmente las capas de refuerzo adheridas, ya que de acuerdo a informe (Rasmussen y Rozychi, 2004), permiten reducir la permeabilidad, el ancho de fisura, y aumentar la resistencia al desgaste. Entre las más comunes se encuentran las sintéticas de polipropileno y poliolefina.

\section{Condiciones climáticas: temperatura y humedad}

Las condiciones climáticas pueden afectar tanto el comportamiento como el rendimiento en una capa de refuerzo con hormigón. El diseño y construcción debe de considerar los cambios en la temperatura de curado y su comportamiento en edades tempranas, así como la susceptibilidad al fenómeno de alabeo y deformaciones. En el caso de las capas de refuerzo más delgadas, estas se exponen más a la posibilidad de afectar las propiedades del hormigón, por lo que la elección del material debe de considerar este tipo de condiciones. La transferencia de cargas debe diseñarse para soportar estos cambios en la temperatura.

Las condiciones climáticas, principalmente la temperatura, es un factor sumamente significativo en el diseño, al igual que el tráfico, siendo claves como dato de entrada en guías de diseño. Por ejemplo, en el caso de la metodología BCOA ME (de la cual se hablará posteriormente), desarrollado por investigadores de la Universidad de Pittsburg y según aclaraciones del señor Robert Rodden, P.E, Director Ejecutivo de la Sociedad Internacional de Pavimentos de Concreto (ISCP, por sus siglas en inglés), la aplicación de este programa de diseño puede adaptarse a condiciones propias de Costa Rica, donde es clave considerar estos factores mencionados anteriormente, dado que influyen significativamente en una adecuada predicción de los resultados.

\section{Metodologías de diseño}

En el diseño de capas de refuerzo se debe verificar dos aspectos de alto grado de importancia para la metodología a utilizar: el primero de ellos, el criterio con el que el método maneja la unión entre el pavimentoasfáltico existente y la capa de hormigón; y el segundo, si el método asume que el pavimento existente provee de forma significativa capacidad estructural o favorece la calidad de fundación del nuevo espesor a colocar (Harrington y Fick, 2014). Entre las metodologías más comunes para el diseño de capas de refuerzo con hormigón adheridas se encuentran (Harrington, Rasmussen y Torres, 2011):

Capas de refuerzo con hormigón sobre asfalto (BCOA, por sus siglas en inglés, 2012). La ACPA (1998) desarrolló un procedimiento mecanicista para diseñar capas de refuerzo delgadas con hormigón (50 mm a $100 \mathrm{~mm}$ ), y de losas pequeñas. El método BCOA es un proceso de diseño iterativo, donde determina la cantidad de camiones permisibles de acuerdo a los datos de espesor, tránsito, módulo de ruptura, entre otros datos ingresados previamente (Harrington y Fick, 2014). En enero de 2011 la ACPA lanzó una aplicación web para el diseño de espesores que incorpora el trabajo de Riley (2006). Este método es válido para losas de espesores entre $75 \mathrm{~mm}$ y $150 \mathrm{~mm}$ y de dimensiones máximas de 1,8m. La actualización de 2012 mejora la incorporación de fibras de refuerzo basándose en los trabajos de Bordelon y Roesler (2012).

Capas de refuerzo con hormigón sobre asfalto, diseño mecánico-empírico (BCOA-ME, Vandenbosshe, 2013). Para el caso de las capas de refuerzo ultradelgadas el que actualmente se está utilizando en ciudades como Chicago es el BCOA ME, de la Universidad de Pittsburgh, según comunicación con el director de la ISCP, para el diseño de este tipo de capas les ha generado buenos resultados. Menciona que el uso de este programa es libre; sin embargo, se encuentra calibrado para condiciones propias de cada uno de los estados del país.

Esta metodología es uno de los avances más recientes en diseño de capas de refuerzo adheridas. En la guía para capas de refuerzo con hormigón (2014), Harrington y Fick explican que este método incorpora el modelo de predicción del ACPA BCOA, prediciendo las grietas de esquina de losas de máximo de 1,35m, y el modelo de comportamiento del Departamento de Transporte de Colorado, determinando el agrietamiento transversal de losas de 3,6m de ancho y un modelo desarrollado recientemente que predice el agrietamiento longitudinal de losas de $1,8 \mathrm{~m}$. 
Entre los principales datos a ingresar se encuentra: tránsito, gradiente equivalente de temperatura (definido por las condiciones locales), latitud, longitud, elevación, datos de la estructura existente como: espesor de la mezcla asfáltica luego del fresado y módulo de reacción de la subrasante. También propiedades de la capa de hormigón, como el módulo elástico, coeficiente de expansión térmica, tipo de fibra a incorporar, etc (Vandenbosshe, Dufalla y Li, 2012). Además la rigidez de la mezcla asfáltica se basa en el diseño de la mezcla, los parámetros utilizados incluyen una gradación estándar y un aglutinante seleccionado con base en las condiciones climáticas regionales del proyecto. El agrietamiento por fatiga en la capa de asfalto antes de colocar la capa de refuerzo también debe ser conocido o estimado.

\section{CONCLUSIONES Y RECOMENDACIONES}

- Ante la presencia de deterioros superficiales en el pavimento asfáltico, la aplicación de capas con hormigón ultradelgadas adheridas se convierte en una alternativa eficiente para la rehabilitación.

- Las capas adheridas actúan monolíticamente con el pavimento existente, disminuyendo la transmisión de esfuerzos tangenciales.
- Una evaluación detallada del pavimento existente es fundamental para determinar si su capacidad estructurales suficiente para soportar las cargas aplicadas. En caso de necesitar de fresado la valoración debe realizarse posterior a la aplicación de esta técnica de reparación previa.

- Un factor clave para el adecuado funcionamiento de las capas de refuerzo ultradelgadas es garantizar una adherencia óptima, por lo que se recomienda verificar su eficacia.

- Las condiciones climáticas, principalmente la temperatura y la predicción del tráfico, requieren de especial valoración en el diseño a realizar de capas de refuerzo.

- La adición de fibras (acero o sintéticas) al hormigón, incrementa significativamente su desempeño, tenacidad y durabilidad; sin embargo, pueden aumentar el costo.

- La metodología BCOA ME es uno de los avances más recientes en diseño de capas de refuerzo adheridas y puede adaptarse a las condiciones propias del país, para lo cual se debe investigar y analizar factores de temperatura y tráfico.

- Tanto el criterio de unión entre el pavimento existente y la capa de refuerzo, como la presencia de capacidad estructural en el pavimento a rehabilitar, son datos necesarios a determinar para la metodología de diseño a utilizar. 


\section{REFERENCIAS BIBLIOGRÁFICAS}

1. Abascal, J. (2013). Refuerzos con hormigón de pavimentos bituminosos. Instituto Español del Cemento y sus Aplicaciones (IECA). Madrid, España.53-1², 11.

2. ASTM D6433-11. (2011). Standard Practice for Roads and Parking Lots Pavement Condition Index Surveys. ASTM International, West Conshocken, PA.doi: 10.1520/D6433-11.

3. Autret, P., yBrousse, J. (1992). VIZIR Método con ayuda de computador para la estimación de necesidades en el mantenimiento de una red carretera. Laboratoire Central des Ponts et Chaussées, Paris, Francia.

4. Barrantes, R., Sanabria, J., Chacón, M., y Loría, G. (2015). Informe de Evaluación de la Red Vial Nacional Pavimentada de CostaRica, Años 2014-2015. Montes de Oca, San José, Costa Rica: Universidad de Costa Rica. INF-PITRA-001-2015, 40-47

5. Bordelon, A., y Roesler, J. (2012). Design with Fiber Reinforcement for Thin Concrete Overlays Bonded to Asphalt. Journal of Transportation Engineering. 430-433. doi: 10.1061/ (ASCE) TE.1943-5436.0000339, 430-435.

6. Coronado, J. (2002). Manual Centroamericano para Diseño de Pavimentos. Secretaría de Integración Económica Centroamericana. USAID/SIECA No. 0596-0184.20. Guatemala.

7. Han, C. (2005). Synthesis of Current Minnesota Practices of Thin and Ultra-Thin Whitetopping. Minnesota Department of Transportation. Research Services Section. MN/RC-2005-27, 1-17.

8. Harrington, D., Rasmussen, R., y Torres, H. (2011). National Concrete Pavement Technology Center. Design of Concrete Overlays Using Existing Methodologies. National Concrete Pavement Technology Center. FHWA, 1-11.

9. Harrington, D.,y Fick, G. (2014). Guide to Concrete Overlays: Sustainable Solutions for Resurfacing and Rehabilitating Existing Pavements. National Concrete Pavement Technology Center. Report No. ACPA TB021.03P. 3rd Edition. Washington, DC.

10. Mack, J.W., Cole, L.W.,y Mohsen, J.P. (1993). Analytical considerations for thin concrete overlays on asphalt. Transportation Research Record No.1388. Washington, D.C.

11. McGhee, K. (1994). Resurfacing. Synthesis of Highway Practice 204: Portland Cement Concrete. Washington, D.C. Transportation Research Board. National Research Council.Washington, D.C.

12. Miller, J., y Bellinger, W. (2003). Distress Identification Manual for the Long-Term Pavement Performance Program. Fourth Revised Edition. Report No. FHWA-RD-03-031. Washington, D.C.

13. Rasmussen, R. O. y D. K. Rozychi. (2004). Thin and Ultra-Thin Whitetopping. NCHRP Synthesis of Highway Practice 338, National Cooperative Highway Research Program, National Research Council Washington, D.C., 3-42.

14. Silfwerbrand, H., Beushausen, H.,y Courard, L. (2011). Bond. En Bissonnette, J. et al. Bonded Cement-Based Material Overlays for the Repair, the Lining or the Strengthening of Slabs or Pavements. RILEM State of the Art Reports. Springer Netherlands. 3(1), 51-79. doi:10.1007/978-94-007-1239-3.

15. Tia, M., Wu, C.L., Tapia, P.,y Kumara, M.A.W. (2007). Evaluation of Feasibility of Using Composite Pavements in Florida by Means of HVS Testing. Department of Civil and Coastal Engineering, College of Engineering, University of Florida, Gainesville, Florida, USA.

16. Trevino, McCullough, F., y Fowler, D. (2003). Techniques and Procedures for Bonded Concrete Overlays. Center of Transportation Research, Report No. FHWA/TX-05/0-4398-2, 21-118.

17. Vandenbosshe, J., N. Dufalla y Z. Li. (2012). A Revised Thin and Ultra-Thin Bonded Whitetopping Design Procedure. Presented at 2013 TRB Annual Meeting.

18. Vandenbossche, J. M.y S. Sachs (2013). Rehabilitation Strategies for Bonded Concrete Overlays of Asphalt Pavements. FHWA Pooled Fund stufy, TPF 5-165.

19. W. K. Mampearachchi, J. V. Kosgolla., y M.Tia. (2012). Feasibility of whitetopping for resurfacing thin asphalt pavements. Journal of the National Science Foundation of Sri Lanka,40(4), 293-302 